\title{
Inteligência Artificial: um desafio na detecção precoce de Indivíduos em Risco de Dislexia
}

\author{
Luís Fernando Orleans ${ }^{1}$, Raimundo José Macário Costa², Sérgio Manoel Serra da \\ Cruz $^{2}$
}
${ }^{1}$ Departamento de Ciência da Computação, Instituto Multidisciplinar - Universidade Federal Rural do Rio de Janeiro
Nova Iguaçu - RJ - Brasil
${ }^{2}$ Departmento de Matemática, Instituto de Ciências Exatas - Universidade Federal Rural do Rio de Janeiro
Seropédica - RJ - Brasil
\{lforleans, macario, serra\}@ufrrj.br

\begin{abstract}
The acquired knowledge on dyslexia can be applied to build intelligent computing systems to be used by both education and health areas. Furthermore, those systems can help dyslexia diagnosis. The purpose of this paper is to point research directions and make considerations on the challenges related to Computational Neuroscience support on the Brazilian educational system. This work also presents a dyslexia based approach which is one of the Learning Disorders that has been attracting attention from both researchers from health and education areas. Finally, this research aims to investigate novel computational techniques to fulfill the lack of data gathering and exploratory analysis - both related to early detection of dyslexic individuals.
\end{abstract}

Resumo. O conhecimento adquirido sobre dislexia pode ser direcionado para a construção de um sistema computacional inteligente aplicado à área de educação e da área de saúde e aliada às novas formas de diagnóstico médico. O objetivo deste artigo é expor direções de pesquisa e considerações sobre os desafios referentes ao apoio computacional oferecido pela Neurociência Computacional na Educação Brasileira. O trabalho também apresenta uma abordagem relacionada com a dislexia, um dos Transtornos de Aprendizagem que tem despertado interesse nos pesquisadores, profissionais de saúde e educação. Esta pesquisa também visa investigar novas técnicas computacionais que venham a preencher a lacuna de ausência de instrumentos de coleta e análise exploratória de dados relacionados à detecção precoce de indivíduos disléxicos.

\section{Introdução}

Há uma diversidade de recursos tecnológicos que podem ajudar pessoas com dificuldades de aprendizagem, sendo que conhecê-los apropriadamente é um desafio para os profissionais desta área de Tecnologia da Informação (TI). Dentre estas dificuldades, podemos exemplificar os seguintes transtornos de aprendizagem: déficit de atenção e hiperatividade (TDAH), dislexia, discalculia, dismusicia, dislalia, entre outros. Se for particularizado para indivíduos disléxicos, as tecnologias que se utilizam para a detecção de indivíduos em risco de dislexia ainda são muito incipientes (SOUSA e SILVEIRA, 2015). 
A detecção precoce das crianças em risco de dislexia é fundamental para a diminuição dos riscos de perda cognitiva e, futuramente, a lacuna escolar certamente será menor. Por outro lado, os baixos níveis sociais e as fragilidades dos vínculos familiares são possibilidades de fatores de risco, acarretando em perdas na linguagem, na memória e nas habilidades sociais, todas de extrema importância para o desenvolvimento cognitivo do indivíduo. Desta forma, com a identificação precoce de crianças em risco de dislexia, os fatores psicológicos e socioafetivos se beneficiam, uma vez que a detecção do problema irá minimizar os sentimentos de fracasso e o desânimo das crianças que enfrentam dificuldades no processo de aprendizagem (CARIDÁ e MENDES, 2012).

Neste sentido, a dislexia pode ser entendida como uma dificuldade enfrentada pelas crianças na idade escolar caracterizando-se como uma deficiência especial na aprendizagem. Esta é compreendida como uma alteração em um ou mais dos processos psicológicos básicos para a compreensão da linguagem escrita ou oral, que pode se manifestar como uma falta de habilidade para leitura, matemática, ou expressão escrita, além de problemas com a memória. Nessas condições podem estar incluídos uma falha de percepção, dano cerebral, disfunção cerebral mínima, dislexia e afasia do desenvolvimento. Não se aplica, assim, a crianças que apresentem deficiências visuais, auditivas, motoras, atraso mental, transtornos emocionais, situações de desvantagem ambiental, cultural ou econômica (SANTOS e MARINHO, 2007).

Dentre os problemas existentes acerca da aprendizagem, a dislexia destaca-se de outros transtornos, com os seus portadores podendo apresentar atrasos graves na leitura, na escrita e ortografia, assim como inversões de símbolos, apresentando natureza única e limitada do déficit fonológico (SHAYWITZ, 1999; MOUSINHO, 2003). Há registros da limitação médica pediátrica para constatar e avaliar os distúrbios de aprendizagem com vistas a um diagnóstico precoce adequado para as crianças com habilidades sociais e desenvolvimento motor apropriados até a terceira série do ensino fundamental.

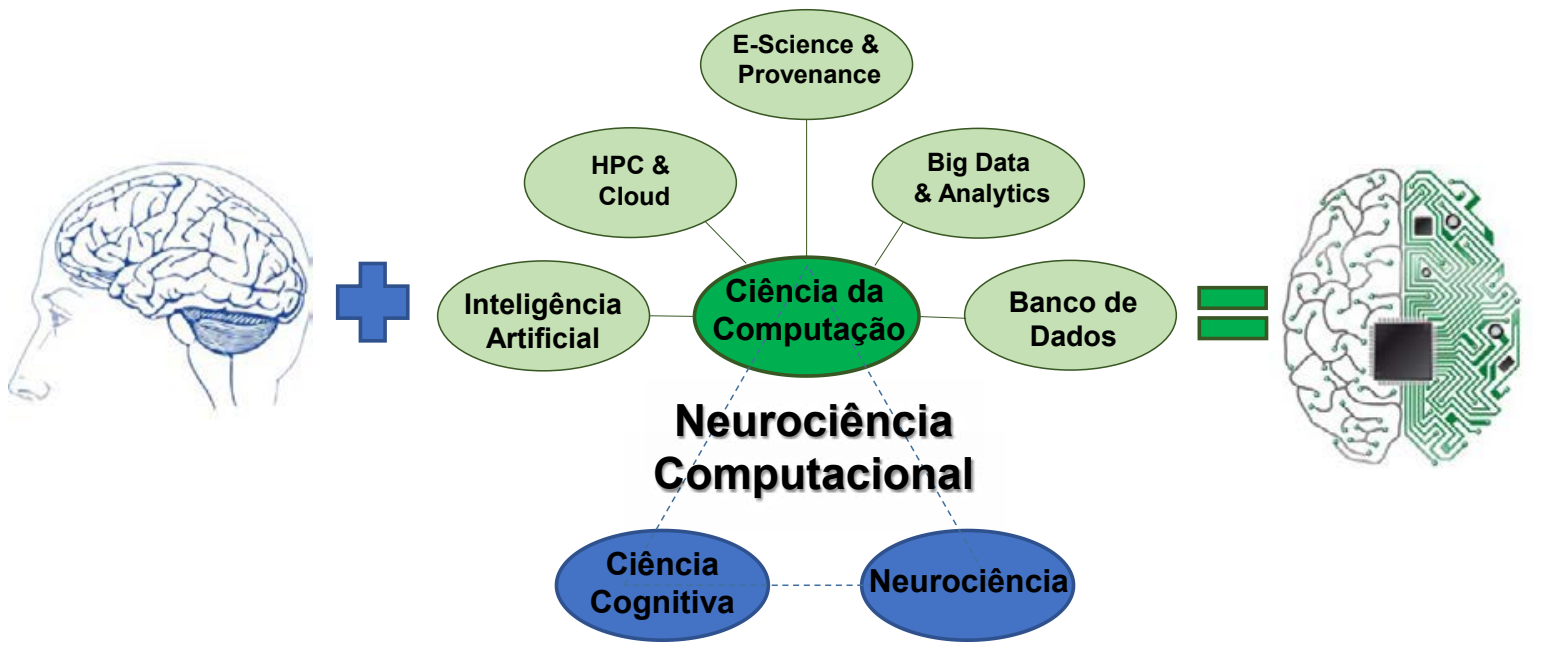

Figura 1. Três pilares na Neurociência Computacional e perspectivas de investigações tecnológicas apoiadas pela Ciência da Computação. Adaptado de Macário Costa et al (2014) 
Um dos caminhos para encarar os desafios e propor soluções para o problema de detecção de indivíduos em risco de dislexia é a Neurociência Computacional (NC) (MACÁRIO COSTA et al, 2014). Esta é uma área essencialmente interdisciplinar e assim está apoiada em três pilares: Neurociência (das áreas de Medicina); Ciência Cognitiva (das áreas de Educação e Psicologia) e Ciência da Computação (áreas de Inteligência Artificial, Bancos de Dados, EScience, Proveniência, Big Data, Nuvens, entre outros) (Figura 1). No entanto, uma das grandes dificuldades desta área é modelar (matemática e computacionalmente) um Transtorno de Aprendizagem, no caso a dislexia, identificar quais são os dados e variáveis mais relevantes, transcrevê-los para as soluções tecnológicas e avaliar se os resultados computacionais são significativos e válidos de acordo com os aspectos médico, ético e educacional.

O objetivo deste artigo é expor direções de pesquisa e considerações sobre os desafios referentes ao apoio computacional oferecido pela Neurociência Computacional na Educação Brasileira. O trabalho também apresenta uma linha de pesquisa relacionada com a dislexia, um dos Transtornos de Aprendizagem que tem despertado interesse nos pesquisadores, profissionais de saúde e educação, e a Computação.

Este artigo está dividido da seguinte forma: a seção 2 caracteriza o transtorno de dislexia, enquanto a seção 3 lista o estado da arte. A seção 4 aborda os desafios no desenvolvimento de sistemas computacionais para detecção precoce de indivíduos em risco de dislexia e, por fim, a seção 5 apresenta as oportunidades em aberto e as considerações finais.

\section{Dificuldades de Aprendizagem e Dislexia}

No âmbito da educação, novos estudos relacionam as dificuldades de aprendizagem enfrentadas por crianças e jovens com diversas técnicas computacionais. Tais questões têm relevância social e podem repercutir na evasão escolar, analfabetismo funcional e sucessivas reprovações. No início do processo de escolarização, a criança pode apresentar algumas dificuldades no aprendizado da leitura, escrita e cálculo. Convencionalmente, costumam-se dividir as dificuldades de aprendizagem em dois tipos: a) Dificuldades Escolares (DE) relacionadas a problemas de origem e ordem pedagógica e b) Distúrbios de Aprendizagem (DA) relacionados a uma disfunção no Sistema Nervoso Central (SNC), caracterizada por uma falha no processo de aquisição e/ou desenvolvimento das habilidades escolares (MACÁRIO COSTA et al. 2014b).

Os DA apresentam comprometimento em um ou mais componentes da linguagem. Trata-se de um transtorno caracterizado por desempenho substancialmente abaixo do esperado (nas áreas da leitura, escrita e matemática) tendo em vista a idade cronológica, medidas de inteligência e educação apropriada para a idade (DSM IV, 1995). Dentre os DA, a dislexia destaca-se de outros transtornos que incluem atrasos graves na leitura, na escrita e ortografia, assim como inversões de símbolos (Anexo I). O destaque se deve a sua natureza única e limitada do déficit fonológico (MOUSINHO, 2003; SHAYWITZ e SHAYWITZ, 1999).

Segundo a Associação Internacional de Dislexia, o transtorno da dislexia é caracterizado pelas dificuldades na correção e/ou fluência na leitura de palavras e por baixa competência leitora e ortográfica. Estas dificuldades resultam tipicamente de um déficit na componente fonológica da linguagem que é frequentemente imprevisto em relação a outras capacidades cognitivas e às condições educativas. Secundariamente, podem surgir dificuldades de compreensão leitora, experiência de leitura reduzida que podem impedir o desenvolvimento 
do vocabulário e dos conhecimentos gerais (Associação Internacional de Dislexia, 2003, cit. por MACÁRIO COSTA et al, 2011).

Quanto ao processo utilizado para a realização do diagnóstico em sua forma tradicional, o responsável pelo paciente recebe um questionário a ser levado para casa e ser respondido. Ao término do preenchimento, o questionário é devolvido ao profissional fonoaudiólogo, que, por sua vez, o confere, certificando que todas as questões foram respondidas. Em muitas vezes, o respondente é questionado sobre algumas das respostas apresentadas. Em outras ocasiões, o profissional deve esclarecer perguntas não compreendidas pelo respondente. Finalmente, em um momento posterior, o questionário é tabulado para verificar o risco ou não de o indivíduo disléxico.

Porém, a confirmação final do diagnóstico de dislexia deverá ser feita por uma equipe multidisciplinar, que será responsável pela elaboração de um processo de avaliação minucioso e abrangente, também conhecido como avaliação multidisciplinar e de exclusão.

Hoje, para fazer uma avaliação de uma pessoa verifica-se uma demora média de três meses para o estabelecimento de um diagnóstico por um profissional qualificado. A partir do estabelecimento de um sistema inteligente pode ser possível que esta demora se reduza a poucas horas, oferecendo oportunidade de atendimento para mais pessoas em um curto espaço, de forma mais oportuna e eficiente.

\section{Estado da Arte}

O conhecimento adquirido na área de neurociência pode ser associado às ferramentas e técnicas computacionais para aperfeiçoar detecção precoce da dislexia. Para alcançarmos um patamar tecnológico compatível com as demandas multifacetadas dos estudos sobre a dislexia, deveremos considerar a estruturação de uma agenda de pesquisa com ênfase em novos modelos e incorporação de técnicas computacionais. Neste caso é possível verificar trabalhos que estão na fronteira da área:

a) Modelagem e desenvolvimento de sistemas inteligentes e preditivos baseados em técnicas de Inteligência Artificial (MACÁRIO COSTA et al, 2007, 2008, 2009, 2010, 2011, 2013; ZAVALETA et al, 2012), os autores apresentam uma implementação de redes neurais para classificar probabilisticamente pacientes jovens e adultos com dislexia. Riveros et al (2005) propõe um sistema especialista voltado para um prédiagnóstico de dislexia em crianças com vista ao desenvolvendo ferramentas lúdicas de avaliação.

b) Desenvolvimento de artefatos de hardwares e softwares como tecnologias assistivas para contornar as dificuldades relacionadas à leitura. Atualmente, existem hardwares do tipo canetas que auxiliam os disléxicos na leitura e reconhecimento de textos. Estas tecnologias não substituem a intervenção direta do profissional de saúde, mas eles podem aumentar a eficácia das terapias (ZONG, 2014).

Essas tecnologias ajudam a aumentar a confiança do indivíduo nos ambientes residencial, sala de aula ou no trabalho. Atualmente já existem softwares no mercado (em língua inglesa) que visam auxiliar indivíduos comprovadamente disléxicos. Por exemplo, o Simon S.I.O, Sortegories, Wordmaker, todos disponíveis no sítio Dyslexia Helper ${ }^{1}$, e

\footnotetext{
${ }^{1} \mathrm{http}: / /$ dyslexiahelp.umich.edu/tools/software-assistive-technology
} 
WebHelpDyslexia ${ }^{2}$. O Simom S.I.O é um tutor pessoal que possibilita que indivíduos disléxicos adquiram as habilidades de leitura. O Sortegories é um plug-in Web que auxilia a construção de vocabulários a partir de fonema básicos e a compreensão de leitura. $\mathrm{O}$ Wordmaker é voltado para estudantes que possuem limitadas habilidades de decodificação, fluência e compreensão de textos, e oferece uma abordagem sistemática e interativa para ensinar ortografia, leitura e escrita para indivíduos disléxicos. O WebHelpDyslexia é um software nacional que permite ao disléxico fazer as alterações necessárias (cor, espaçamento, tamanho de fonte) para visualizar e melhorar o entendimento de um texto sendo lido. $\mathrm{O}$ software também realça partes do texto, por meio de uma "régua de leitura", o que reduz a distração.

\section{Desafios Existentes}

Dentro do contexto computacional, a Inteligência Artificial (IA) estuda processos cognitivos e tenta criar sistemas computacionais situando-se na fronteira entre diversas ciências tais como Psicologia, Filosofia, Linguística e Engenharia. A Inteligência Artificial pode desempenhar um papel importante na busca, elaboração e validação de modelos computacionais na construção de sistemas adaptativos e/ou evolutivos inteligentes (CARVALHO, 2005). É importante frisar a escassez de informações disponíveis sobre sistemas computacionais voltados para o rastreio de pessoas com sinais de dislexia e outros transtornos. Dessa forma a lacuna de conhecimento a ser explorado justifica o investimento nacional em pesquisas e desenvolvimento de tecnologias.

De acordo com o exposto acima é admissível a criação de modelos computacionais inteligentes capazes de rastrear grupos de padrões por similaridades. Com as técnicas de Inteligência Artificial espera-se desenvolver um sistema computacional que seja capaz de rastrear pessoas em risco de dislexia.

Para alcançarmos um patamar tecnológico compatível com as demandas multifacetadas dos estudos do cérebro no Século XXI, deveremos considerar a estruturação de uma agenda de pesquisa com ênfase em novos modelos e incorporação de técnicas computacionais (intensivas em dados) do E-science (HEY et al, 2009) para o desenvolvimento de aplicações em Neurociência Computacional. Neste caso é possível avançar nas seguintes áreas:

a) Desenvolvimento de sistemas inteligentes e preditivos baseados em técnicas de Inteligência Artificial (MACÁRIO COSTA et al, 2007, 2008, 2009, 2010, 2011, 2013; ZAVALETA et al, 2012) capazes de manipular grandes volumes de dados;

b) Uso ambientes computacionais distribuídos de processamento de alto desempenho para apoiar simulações e experimentos in silico baseados em workflows científicos (DEELMAN et al, 2009) de simulações de modelos cerebrais (ABBOT, 2013; KUBILIUS, 2014; NIH, 2014; HBP, 2014).

c) Adoção de técnicas de gestão de grandes volumes de dados biológicos semiestruturados e processamentos típicos de Big Data (DAVISON, 2010; BERMAN, 2011; ABBOTT, 2013), os projetos de neurociência computacional tendem mapear modelos cerebrais cada vez maiores, mais complexos e utilizar sensores e dados com diversos formatos (ZHONG et al. 2011; ZHONG, 2014);

\footnotetext{
${ }^{2}$ http://ead.unifal-mg.edu.br/cead/?q=node/194
} 
d) Incorporação de descritores de proveniência (CRUZ et al, 2009) e curadoria de dados e técnicas de gestão de conhecimento para ampliar a reprodutibilidade e a confiabilidade dos estudos em neurociência computacional (CHEN, ZHONG, LIANG, 2012; CICCARESE et al, 2013) estes tendem a ser conduzidos por times de pesquisa interdisciplinares e dispersos geográfica e temporalmente (CHEN, ZHONG, 2013);

O conhecimento adquirido na área de Neurociência Computacional pode ser associado às ferramentas e técnicas computacionais para aperfeiçoar as oportunidades de atuação sobre Transtornos de Aprendizagem.

\section{Considerações Finais}

Identificar precocemente crianças com dificuldades de leitura e aprendizagem torna-se prioridade diante da possibilidade de poder eliminar ou minimizar prejuízos escolares e sociais nas mesmas. Dessa maneira, as estratégias computacionais da Neurociência Computacional podem fazer toda a diferença nessa identificação.

Esta proposta de desafio visa investigar novas técnicas computacionais que venham a preencher a lacuna de ausência de instrumentos de coleta e análise exploratória de dados relacionados à detecção precoce de disléxicos em idade escolar no Brasil.

\section{Agradecimentos}

Os autores agradecem à FAPERJ e ao MEC/SeSU pelo apoio financeiro a pesquisa.

\section{REFERÊNCIAS}

ABBOTT, A. (2013) “Neuroscience: Solving the brain”, In: Nature 499, pages. 272-274.

BOULIC, R. and RENAULT, O. (1991) "3D Hierarchies for Animation”, In: New Trends in Animation and Visualization, Edited by Nadia Magnenat-Thalmann and Daniel Thalmann, John Wiley \& Sons ltd., England.

CARIDÁ, Déborah Alcântara Prósperi; MENDES, Mônica Hoehne. “A importância do estímulo precoce em casos com risco para dislexia: um enfoque psicopedagógico". Rev. Psicopedagogia 2012; 29(89): 226-35. Acesso em 15/04/2016.

CARVALHO, L. A. V., 2005, Datamining - A Mineração de Dados no Marketing, Medicina, Economia, Engenharia e Administração. Rio de Janeiro: Editora Ciência Moderna Ltda.

CICCARESE, P., SOILAND-REYES, S., BELHAJJAME, K., GRAY, A.J.G, GOBLE, C., Clark, T. (2013) "PAV ontology: provenance, authoring and versioning", Journal of Biomedical Semantics 2013, 4:37.

CHEN J. H., ZHONG, N. (2013) "Toward the Data-Brain driven systematic brain data analysis". IEEE Transactions on Systems, Man, and Cybernetics: Systems, 43(1), pages. 222-228.

CHEN J. H., ZHONG, N., LIANG, P. P. (2012) "Data-Brain driven systematic human brain data analysis: A case study in numerical inductive reasoning centric investigation" Cognitive Systems Research, Elsevier, vol. 15-16, pages. 17-32.

CRUZ, S.M.S, CAMPOS, M. L M., MATTOSO, M. (2009) "Towards a Taxonomy of Provenance in Scientific Workflow Management Systems". SERVICES I 2009: 259-266. 
DAVISON, A.P. (2010) "Challenges and solutions in replicability and provenance tracking for simulation projects". BMC Neuroscience 2010, 11(Suppl 1):P76.

DEELMAN E, Gannon D, SHIELDS M, Taylor I (2009) "Workflows and e-Science: An overview of workflow system features and capabilities", Future Generation Computer Systems 25(5):528-540.

DUTRA, M. V. O.; SOUZA, M. N., 2001, Redes neurais artificiais e análise fatorial no diagnóstico do distúrbio neuropsicológico infantil. Memorias II Congresso Latinoamericano de Ingenieria Biomédica, Habana. La Habana, Cuba.

DSM-IV, 1995, Manual diagnóstico e estatístico de transtornos mentais. Porto Alegre: Artes Médicas. Trad. da edição da APA. Diagnostic and Statical Manual of Mental Disorders, 4 ed., 1994.

GARCIA F.B. et al, 2008. Importância dos testes sorológicos de triagem e confirmatórios na detecção de doadores de sangue infectados pelo vírus da hepatite C. Revista brasileira de hematologia e hemoterapia. 2008;30(3):218-222. Acesso julho 2014.

HBP (2014) "Human Brain Project”. https://www.humanbrainproject.eu/

HEY, T., Tansley, S., Tolle, K (2009) "The Fourth Paradigm: Data-Intensive Scientific Discovery" Microsoft Press. 1st Edition.

KUBILIUS, J. (2013) "A framework for streamlining research workflow in neuroscience and psychology". Front. Neuroinform. 7: 52.

LOPES CK, OLIVEIRA CI. A dislexia na ótica do psicopedagogo. 2007. Acesso em: $15 / 04 / 2016$.

MACÁRIO COSTA, R. J. et al.(2014) "Desafios e Oportunidades em Neurociência Computacional na Educação Brasileira". In: Grandes Desafios da Computação no Brasil. $3^{\circ}$ Seminário. SBC, 2014a.

MACÁRIO COSTA, R. J. et al.(2014) "Usando Tecnologias de Neurociência Computacional na Educação Brasileira”. In: Conferência Internacional sobre Informática na Educação, 2014, Fortaleza, XIX TISE, 2014 b.

MACÁRIO COSTA, R. J. et al. Classificação de pacientes com transtorno de dislexia usando redes neurais artificiais. In: Congresso Nacional de Matemática Aplicada e Computacional, 2007, Florianópolis. XXX CNMAC. Florianópolis, 2007

MACÁRIO COSTA, R. J. ; MOUSINHO, Renata ; VIDAL, L. A. . Redes Neuronais: um instrumento no rastreio (screening) de pessoas com risoco de transtorno específico de leitura. In: I Congresso Ibro/Larc de Neurociências da América Latina, Caribe e Península Ibérica, 2008, Búzios - RJ. Anais do I Congresso Ibro/Larc de Neurociências, 2008.

MACÁRIO COSTA, R. J. ; MOUSINHO, Renata; VIDAL, L. A. . Abordagem Computacional no Screening da Dislexia e do TDAH. In: XXXII Congresso Nacional de Matemática Aplicada e Computacional, 2009, Cuiabá - MT. Anais do CNMAC 2009A.

MACÁRIO COSTA, R. J.; MOUSINHO, RENATA ; VIDAL, L. A. . Dislexia e Inteligência Computacional: Um sistema para rastrear (Screening) pessoas com sinais de transtorno de leitura. In: 2o. Congresso Internacional de Dislexia, 2009, São Paulo. Anais do II Congresso Internacional de Dislexia, 2009B.

MACÁRIO COSTA, R. J. et al. Redes neuronais e transtornos de aprendizagem: rastreio de pessoas com dislexia. In: Simpósio Brasileiro de Informática na Educação, 2009, 
Florianopólis. Anais do Simpósio Brasileiro de Informática na Educação, 2009C. v. 20. p. $1-10$.

MACÁRIO COSTA, R. J. et al. Abordagem tecnológica para rastreio de pessoas com dislexia. Tecer (Belo Horizonte), v. 4, p. 41-53, 2011 A.

MACÁRIO COSTA, R. J. Uma Estratégia computacional na detecção da dislexia. Rio de Janeiro: Tese - UFRJ/COPPE/Programa de Engenharia de Sistemas e Computação, 2011B.

MACÁRIO COSTA, R. J. et al. A Computational Approach for Screening Dyslexia.. In: CBMS 2013, 2013, Porto. 26th IEEE International Symposium on Computer-Based Medical Systems, 2013.

MOUSINHO, R. (2003) "Desenvolvimento da Leitura, Escrita e seus Transtornos. In: Goldfeld, M. Fundamentos em Fonoaudiologia”. Guanabara Koogan. 2a edição.

NIH (2014) “BRAIN 2025: A Scientific Vision”, http://www.nih.gov/science/brain/2025/.

OLIVETTE, T. W. 2009. Diagnóstico Etiológico de AVC Isquêmico em Pacientes Joinvilenses utilizando Redes Neurais Artificiais. Joinvile. SC.

RIVEROS, L. J. M., SOARES, F. S. \& MUNZLINGER, E. Validade de um Sistema Especialista para o Pré-Diagnóstico da Dislexia. XXV Congresso da Sociedade Brasileira de Computação. UNISINOS - São Leopoldo / RS. 22 a 29 de julho. 2005.

SANTOS, C.F.L. e MARINHO, D.H., 2007, Reconhecimento e manejo do distúrbio de leitura na criança. Monografia. (Aperfeiçoamento/Especialização em Residência em Pediatria) Hospital Universitário Evangélico de Curitiba.

SHAYWITZ, S., 2006, Entendendo a dislexia: um novo e completo programa para todos os níveis de problemas de leitura; tradução Vinicius Figueira. - Porto Alegre : Artmed.

SHAYWITZ, S.E. SHAYWITZ, B.A. (1999) "Dyslexia In: Swaiman KF, Ashwal S. Pediatric Neurology - Principal e Practice", Connecticut. Ed. Mosby

SOUSA, Leonardo Ramon Nunes de; Silveira, Ismar Frango. "Desafios das Interfaces para a Aprendizagem de Pessoas com Dispraxia”, 2015. DesafIE 2015 CSBC. Acesso em 15/04/2016.

ZAVALETA, J. et al. (2012) "DysDTool: Uma Ferramenta Inteligente para Avaliação e Intervenção no Apoio ao Diagnóstico da Dislexia”. In: CSBC- XII Workshop de Informática Médica.

ZHONG, N. et al. (2011) “Brain Informatics”. IEEE Intelligent Systems. September, pages 1620.

ZHONG, N (2014) "Research Issues and Challenges on Brain Informatics Towards Computing \& Intelligence in the Big Data Era". In: Pacific-Asia Conference on Knowledge Discovery and Data Mining, $18^{\text {th }}$ PAKDD, 2014. 


\section{ANEXO I -Dificuldades Características da Dislexia}

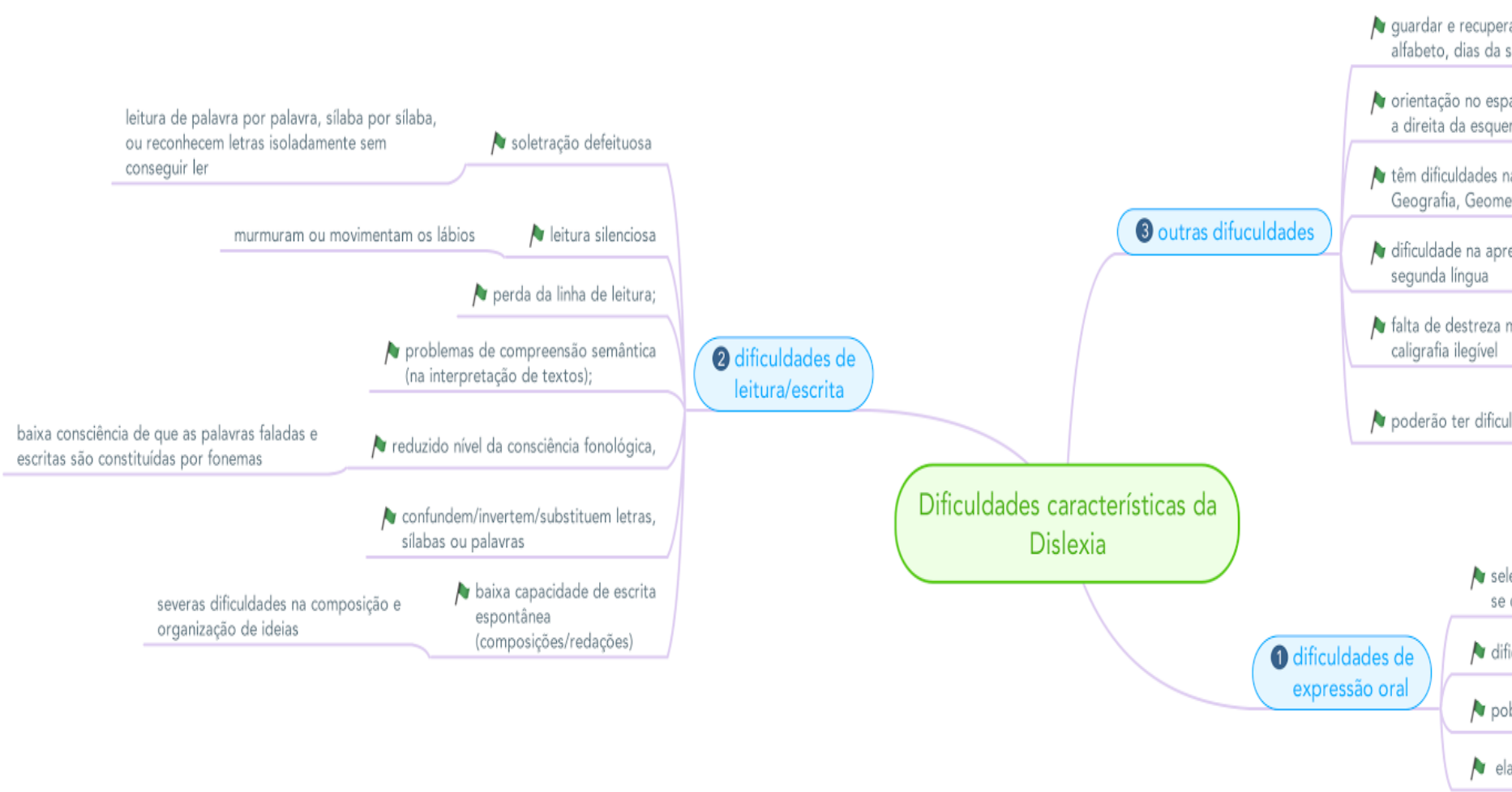

